\title{
FINITENESS THEOREMS ON BLOW-NASH TRIVIALITY FOR REAL ALGEBRAIC SINGULARITIES
}

\author{
SATOSHI KOIKE \\ Department of Mathematics, Hyogo University of Teacher Education \\ Shimokume 942-1, Yashiro, Kato, Hyogo 673-1494, Japan \\ E-mail: koike@sci.hyogo-u.ac.jp
}

When we consider the classification problem of real singularities, it is important to show triviality theorems. Then, for a question whether the triviality we consider is suitable or not, it seems to be natural to ask if a finiteness theorem (or a local finiteness theorem) holds on the triviality. We first ask a finiteness theorem for a family of real algebraic sets.

For a family of algebraic sets, finiteness theorems have been established on topological triviality by T. Fukuda $[\mathrm{Fu}]$ and A. N. Varchenko $[\mathrm{V}]$ and, more strongly, a finiteness theorem for a family of semialgebraic sets on semialgebraic triviality by R. M. Hardt [Ha]. Besides them, finiteness theorems on topological triviality are known for a family of polynomial function-germs $([\mathrm{Fu}])$ and for a family of two-variable polynomial map-germs (K. Aoki [A], C. Sabbah [Sa-1]). On the other hand, it is also known that local topological moduli appear in a family of three-variable polynomial map-germs (I. Nakai $[\mathrm{N}]$ ).

Here we consider the following problem:

Does a finiteness theorem hold for stronger triviality in a structural meaning?

Let $F(x ; t)$ be a family of polynomial mappings. Then we can describe the problem above more explicitly. Namely, we ask the following. After a finite subdivision of the parameter space, do there exist a desingularisation of the algebraic variety defined by $F=0$ and some triviality upstairs which induces a topological (or semialgebraic) trivialisation of the zero-set $F^{-1}(0)$ ?

If the trivialisation upstairs is a regular isomorphism, we say that the family of zero-

2000 Mathematics Subject Classification: 14P20, 58C27, 14P25, 57R45, 58A07, 58A35.

Key words and phrases: Blow-Nash Triviality, Simultaneous Resolution, Semialgebraicity, Isotopy Lemma.

Research of the author supported by Grant-in-Aid for Scientific Research (No. 13640070) of the Ministry of Education, Science and Culture of Japan, and a grant of the Japan Association for Mathematical Sciences.

The paper is in final form and no version of it will be published elsewhere. 
sets $F^{-1}(0)$ admits a Blow-regular trivialisation, following Tzee-Char Kuo's terminology. He introduced the notion of blow-analytic equivalence for real analytic function-germs ([Ku-1], [Ku-2], [Ku-3], [FKK]).

We recall the Whitney function $([\mathrm{W}])$. Let $J=(1, \infty)$ and let $f_{t}:\left(\mathbf{R}^{2}, 0\right) \rightarrow(\mathbf{R}, 0)$, $t \in J$, be a function defined by

$$
f_{t}(x, y)=x y(x-y)(x-t y) .
$$

Then the zero-set consists of 4 lines intersecting at the origin of $\mathbf{R}^{2}$ for each $t$.

Define $F:\left(\mathbf{R}^{2} \times J,\{0\} \times J\right) \rightarrow(\mathbf{R}, 0)$ by $F(x, y ; t)=f_{t}(x, y)$. Then it is easy to see that $\left(\mathbf{R}^{2} \times J, F^{-1}(0)\right)$ is topologically trivial. But a finiteness theorem does not hold for $\left(\mathbf{R}^{2} \times J, F^{-1}(0)\right)$ on Blow-regular triviality. Namely, $\left(\mathbf{R}^{2} \times J_{0}, F^{-1}(0)\right)$ is not Blowregularly trivial over any open subinterval $J_{0}$ of $J$, taking an arbitrary finite subdivision of $J$. This follows from the fact that a regular automorphism of $P^{1}(\mathbf{R})$ is linear (see Appendix in T. Fukui, S. Koike and M. Shiota [FKS]). Therefore Blow-regular triviality does not answer our purpose. It is too strong even for a family of plane algebraic curves with isolated singularities. On the other hand, $\left(\mathbf{R}^{2} \times J, F^{-1}(0)\right)$ admits a $\Pi$-Blow-Nash trivialisation along $J$ ([K-2]). Here $\Pi=\pi \times \operatorname{id}_{J}$ where $\pi$ is a blowing-up at $0 \in \mathbf{R}^{2}$. Taking these observations into consideration, let us consider everything in the Nash category after this.

1. Some properties on semialgebraicity and Nash. We first recall the definitions of a Nash manifold and a Nash mapping. Let $r=1,2, \ldots, \infty, \omega$. A semialgebraic set of $\mathbf{R}^{m}$ is called a $C^{r}$ Nash manifold, if it is a $C^{r}$ submanifold of $\mathbf{R}^{m}$. In this paper, a submanifold always means a regular submanifold. Let $M \subset \mathbf{R}^{m}$ and $N \subset \mathbf{R}^{n}$ be $C^{r}$ Nash manifolds. A $C^{s}$ mapping $f: M \rightarrow N(s \leq r)$ is called a $C^{s}$ Nash mapping, if the graph of $f$ is semialgebraic in $\mathbf{R}^{m} \times \mathbf{R}^{n}$.

We next recall some important results on semialgebraicity and Nash.

Theorem 1.1 (B. Malgrange $[\mathrm{M}]$ ).

(1) $A C^{\infty}$ Nash manifold is a $C^{\omega}$ Nash manifold.

(2) A $C^{\infty}$ Nash mapping between $C^{\omega}$ Nash manifolds is a $C^{\omega}$ Nash mapping.

After this, a Nash manifold and a Nash mapping mean a $C^{\omega}$ Nash manifold and a $C^{\omega}$ Nash mapping, respectively. We call the zero-set of a Nash mapping a Nash set.

Theorem 1.2 (Tarski-Seidenberg Theorem [Se]). Let A be a semialgebraic set in $\mathbf{R}^{k}$, and let $f: \mathbf{R}^{k} \rightarrow \mathbf{R}^{m}$ be a semialgebraic mapping, namely, the graph of $f$ is semialgebraic in $\mathbf{R}^{k} \times \mathbf{R}^{m}$. Then $f(A)$ is semialgebraic in $\mathbf{R}^{m}$.

Theorem 1.3 (Łojasiewicz's Semialgebraic Triangulation Theorem [L-1], [L-2]). Given a finite system of bounded semialgebraic sets $X_{\alpha}$ in $\mathbf{R}^{n}$, there exist a simplicial decomposition $\mathbf{R}^{n}=\bigcup_{a} C_{a}$ and a semialgebraic automorphism $\tau$ of $\mathbf{R}^{n}$ such that

(1) each $X_{\alpha}$ is a finite union of some of the $\tau\left(C_{a}\right)$,

(2) $\tau\left(C_{a}\right)$ is a Nash manifold in $\mathbf{R}^{n}$ and $\tau$ induces a Nash diffeomorphism $C_{a} \rightarrow$ $\tau\left(C_{a}\right)$, for every a. 
REMARK 1.4. In Theorem 1.3, the boundedness is not essential. In fact, there is a Nash embedding of $\mathbf{R}^{n}$ into $\mathbf{R}^{n+1}$ via $\mathbf{R}^{n} \subset S^{n}$. Then every semialgebraic set in $\mathbf{R}^{n}$ can be considered as a bounded semialgebraic set in $\mathbf{R}^{n+1}$.

Theorem 1.5 (Shiota's Approximation Theorem [S-2], [S-3], [S-1]). Let $M_{1} \subset M$ be Nash manifolds such that $M_{1}$ is closed in $M$, let $N$ be a Nash manifold and let $f: M \rightarrow N$ be a $C^{r}$ Nash mapping, $r<\infty$, such that $\left.f\right|_{M_{1}}$ is of Nash class. Then we can approximate $f$ by a Nash mapping $g$ in the $C^{r}$ topology so that $f=g$ on $M_{1}$.

For the definition of $C^{r}$ topology, see [S-3].

In general, a Nash equivalence is stronger than a $C^{\omega}$ equivalence. In fact, M. Shiota ([S-3]) showed that there are affine nonsingular algebraic varieties $V_{1}$ and $V_{2}$ which are $C^{\omega}$ equivalent but not Nash equivalent.

2. Nash simultaneous resolution. Let $M, U$ be Nash manifolds, and let $V$ be a Nash set of $U$. Let $\Pi: M \rightarrow U$ be a proper Nash modification. We say that $\Pi$ is a Nash resolution of $V$ in $U$, if there is a finite sequence of blowings-up $\sigma_{j+1}: M_{j+1} \rightarrow M_{j}$ with smooth centres $C_{j}$ (where $\sigma_{j}, M_{j}$ and $C_{j}$ are of Nash class) such that:

(1) $\Pi$ is the composite of $\sigma_{j}$ 's.

(2) The critical set of $\Pi$ is a union of Nash divisors $D_{1}, \ldots, D_{d}$.

(3) $V^{\prime}$ (the strict transform of $V$ in $M$ by $\Pi$ ) is a Nash submanifold of $M$.

(4) $V^{\prime}, D_{1}, \ldots, D_{d}$ simultaneously have only normal crossings.

(5) There is a thin Nash (or semialgebraic) set $T$ in $V$ so that $\left.\Pi\right|_{\Pi^{-1}(V-T)}$ : $\Pi^{-1}(V-T) \rightarrow V-T$ is a Nash isomorphism.

Let $V$ be a Nash set of a Nash manifold $M \subset \mathbf{R}^{m}$. Concerning a Nash resolution, we have the following theorem.

Theorem 2.1 ([H-1], [H-3], [BM-1], [BM-2], [BM-3])). For a Nash variety $V$ of a Nash manifold $U$, there exists a Nash resolution of $V$ in $U, \Pi: M \rightarrow U$.

Let $\mathcal{M}, \mathcal{U}, I$ be Nash manifolds, and let $\mathcal{V}$ be a Nash set of $\mathcal{U}$. Let $\Pi: \mathcal{M} \rightarrow \mathcal{U}$ be a proper Nash modification, and let $q: \mathcal{U} \rightarrow I$ be an onto Nash submersion. For $t \in I$, we set $U_{t}=q^{-1}(t), V_{t}=\mathcal{V} \cap U_{t}$ and $M_{t}=(q \circ \Pi)^{-1}(t)$. We say that $\Pi$ gives a Nash simultaneous resolution of $\mathcal{V}$ in $\mathcal{U}$ over $I$, if there is a finite sequence of blowings-up $\tilde{\sigma}_{j+1}: \mathcal{M}_{j+1} \rightarrow \mathcal{M}_{j}$ with smooth centres $\tilde{C}_{j}$ (where $\tilde{\sigma}_{j}, \mathcal{M}_{j}$ and $\tilde{C}_{j}$ are of Nash class) such that:

(1) $\Pi$ is the composite of $\tilde{\sigma}_{j}$ 's.

(2) The critical set of $\Pi$ is a union of Nash divisors $\mathcal{D}_{1}, \ldots, \mathcal{D}_{d}$.

(3) $\mathcal{V}^{\prime}$ (the strict transform of $\mathcal{V}$ in $\mathcal{M}$ by $\Pi$ ) is a Nash submanifold of $\mathcal{M}$.

(4) $\mathcal{V}^{\prime}, \mathcal{D}_{1}, \ldots, \mathcal{D}_{d}$ simultaneously have normal crossings. The restrictions

- $\left.q \circ \Pi\right|_{\mathcal{V}^{\prime}}: \mathcal{V}^{\prime} \rightarrow I$,

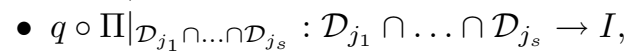

- $\left.q \circ \Pi\right|_{\mathcal{V}^{\prime} \cap \mathcal{D}_{j_{1}} \cap \ldots \cap \mathcal{D}_{j_{s}}}: \mathcal{V}^{\prime} \cap \mathcal{D}_{j_{1}} \cap \ldots \cap \mathcal{D}_{j_{s}} \rightarrow I\left(1 \leq j_{1}<\ldots<j_{s} \leq d\right)$

are onto submersions. 
(5) There is a thin Nash (or semialgebraic) set $\mathcal{T}$ in $\mathcal{V}$ so that $\mathcal{T} \cap V_{t}$ is a thin set in $V_{t}$ for each $t \in I$, and that $\left.\Pi\right|_{\Pi^{-1}(\mathcal{V}-\mathcal{T})}: \Pi^{-1}(\mathcal{V}-\mathcal{T}) \rightarrow \mathcal{V}-\mathcal{T}$ is a Nash isomorphism.

We can define the notion of Nash simultaneous resolution in the local case similarly. Let $I$ be a Nash manifold, and let $F:\left(\mathbf{R}^{n} \times I,\{0\} \times I\right) \rightarrow\left(\mathbf{R}^{p}, 0\right)$ be a Nash map-germ. We say that a Nash modification $\Pi: \mathcal{M} \rightarrow \mathbf{R}^{n} \times I$ gives a Nash simultaneous resolution of $V=F^{-1}(0)$ in $\mathbf{R}^{n} \times I$ over $I$ around $\{0\} \times I$, if there are open Nash submanifolds $\mathcal{N}, \mathcal{U}$ with

$$
\Pi^{-1}(\{0\} \times I) \subset \mathcal{N} \subset \mathcal{M} \text { and }\{0\} \times I \subset \mathcal{U} \subset \mathbf{R}^{n} \times I
$$

such that $\left.\Pi\right|_{\mathcal{N}}: \mathcal{N} \rightarrow \mathcal{U}$ gives a Nash simultaneous resolution of $F^{-1}(0) \cap \mathcal{U}$ in $\mathcal{U}$ over $I$.

3. Nash Isotopy Lemma. Let $M$ be a Nash manifold possibly with boundary, and let $N_{1}, \ldots, N_{b}$ be Nash submanifolds of $M$ possibly with boundary which together with $N_{0}=\partial M$ are normal crossing. Assume that $\partial N_{i} \subset N_{0}, i=1, \ldots, b$. Then we have

Theorem 3.1 (Nash Isotopy Lemma [FKS]). Let $\varpi: M \rightarrow \mathbf{R}^{k}$ be a proper onto Nash submersion such that for $0 \leq i_{1}<\ldots<i_{s} \leq b$,

$$
\left.\varpi\right|_{N_{i_{1}} \cap \ldots \cap N_{i_{s}}}: N_{i_{1}} \cap \ldots \cap N_{i_{s}} \rightarrow \mathbf{R}^{k}
$$

is also a proper onto submersion. Then there is a Nash diffeomorphism

$$
\phi:\left(M ; N_{1}, \ldots, N_{b}\right) \rightarrow\left((M)_{0} ;\left(N_{1}\right)_{0}, \ldots,\left(N_{b}\right)_{0}\right) \times \mathbf{R}^{k}
$$

such that $\varpi \circ \phi^{-1}:(M)_{0} \times \mathbf{R}^{k} \rightarrow \mathbf{R}^{k}$ is the canonical projection. Here $(Z)_{0}$ denotes $\left(\left.\varpi\right|_{Z}\right)^{-1}(0)$ for a subset $Z \subset M$.

REMARK 3.2. From the proof of the theorem above, we have the following.

(1) We can replace $\mathbf{R}^{k}$ by a Nash manifold which is Nash diffeomorphic to an open simplex in a Euclidean space.

(2) The $\phi$ Nash-trivialises not only $M, N_{1}, \ldots, N_{b}$ but also their arbitrary intersections.

The integration of a vector field is a regular method to show $C^{\infty}$ triviality (more generally, $C^{r}$ triviality) or $C^{\omega}$ triviality for a family of sets or mappings. But the integration of a Nash vector field is not necessarily of Nash class (cf. 2.1 in [FKS]). In such sense, the Isotopy Lemma above is an effective tool to show Nash triviality. Although the integration method is not useful in the Nash category, we have instead a powerful tool called Shiota's Approximation Theorem (cf. Theorem 1.5). In fact, the Approximation Theorem takes a very important role in the proof of our Nash Isotopy Lemma.

Nash Isotopy Lemma was first proved by M. Coste and M. Shiota [CS-1] for only a Nash manifold $M$ without boundary, and then proved also for a Nash manifold $M$ with boundary in [CS-2]. Using them, the Isotopy Lemma was shown for a pair of a Nash manifold $M$ and its Nash submanifold $N$ without boundary ([K-2]). The above lemma treats the normal crossing case, containing all the results. The reader would be able to guess that our Nash Isotopy Lemma will be applied to show the Nash triviality of the Nash simultaneous resolution described in the former section. 
4. Nash trivial simultaneous resolution. Let $\Pi: \mathcal{M} \rightarrow \mathcal{U}$ be a Nash simultaneous resolution of a Nash variety $\mathcal{V}=\{F=0\}$ in $\mathcal{U}$ over $I$ in the sense of Section 2. Let $t_{0} \in I$. We say that $\Pi$ gives a Nash trivial simultaneous resolution of $\mathcal{V}$ in $\mathcal{U}$ over $I$, if there is a Nash diffeomorphism $\phi: \mathcal{M} \rightarrow M_{t_{0}} \times I$ such that

(1) $(q \circ \Pi) \circ \phi^{-1}: M_{t_{0}} \times I \rightarrow I$ is the natural projection,

(2) $\phi\left(\mathcal{V}^{\prime}\right)=V_{t_{0}}^{\prime} \times I, \phi\left(\mathcal{D}_{j_{1}} \cap \ldots \cap \mathcal{D}_{j_{s}}\right)=\left(D_{j_{1}, t_{0}} \cap \ldots \cap D_{j_{s}, t_{0}}\right) \times I, \phi\left(\mathcal{V}^{\prime} \cap \mathcal{D}_{j_{1}} \cap \ldots \cap \mathcal{D}_{j_{s}}\right)=$ $\left(V_{t_{0}}^{\prime} \cap D_{j_{1}, t_{0}} \cap \ldots \cap D_{j_{s}, t_{0}}\right) \times I\left(1 \leq j_{1}<\ldots<j_{s} \leq d\right)$.

In the local case, we define the notion of a Nash trivial simultaneous resolution of $F^{-1}(0)$ in $\mathbf{R}^{n} \times I$ over $I$ around $\{0\} \times I$ similarly. Here, we may require also the condition that $\phi\left(\Pi^{-1}(\{0\} \times I)\right)=\pi_{t_{0}}^{-1}(0) \times I$, for our finiteness property.

We now prepare some notation. Let $f_{t}: X \rightarrow Y(t \in J)$ be a mapping. Define a mapping $F: X \times J \rightarrow Y$ by $F(x ; t)=f_{t}(x)$. For a subset $Q \subset J$, set $F_{Q}=\left.F\right|_{X \times Q}$. We use the notation throughout this paper.

We first consider the compact case. Let $N$ be a compact Nash manifold, and let $J$ be a semialgebraic set in some Euclidean space. Let $f_{t}: N \rightarrow \mathbf{R}^{k}(t \in J)$ be a Nash mapping. Assume that $F$ is a Nash mapping. Set

$$
K=\left\{t \in J \mid f_{t}^{-1}(0) \cap S\left(f_{t}\right) \text { is isolated }\right\} .
$$

Here $S\left(f_{t}\right)$ denotes the singular points set of $f_{t}$. It is easy to see that $K$ is a semialgebraic subset of $J$, using Hardt's semialgebraic triviality theorem.

We have the following finiteness theorem on Nash trivial simultaneous resolution.

ThEOREM Ia $([\mathrm{K}-3])$. There exists a finite partition

$$
J=Q_{1} \cup \ldots \cup Q_{s} \cup Q_{s+1} \cup \ldots \cup Q_{u}
$$

with $K=Q_{1} \cup \ldots \cup Q_{s}$ and $J-K=Q_{s+1} \cup \ldots \cup Q_{u}$ which satisfies the following conditions:

(1) Each $Q_{i}$ is a Nash manifold which is Nash diffeomorphic to an open simplex in some Euclidean space.

(2) For each $i$, there is a Nash trivial simultaneous resolution $\Pi_{i}: \mathcal{M}_{i} \rightarrow N \times Q_{i}$ of $F_{Q_{i}}^{-1}(0)$ in $N \times Q_{i}$ over $Q_{i}$.

In particular, for $1 \leq i \leq s$, this Nash trivialisation induces the semialgebraic trivialisation of $F_{Q_{i}}^{-1}(0)$ in $N \times \bar{Q}_{i}$ over $Q_{i}$. (In this case, we say that $\left(N \times Q_{i}, F_{Q_{i}}^{-1}(0)\right)$ admits a $\Pi_{i}$-Blow-Nash trivialisation along $Q_{i}$.)

Outline of the proof. Let $\operatorname{dim} J=r$.

(1) By Hardt's theorem, there is a finite partition of $J$ into semialgebraic sets $R_{k}$ such that $\left(N \times R_{k}, F_{R_{k}}^{-1}(0)\right)$ is semialgebraically trivial over each $R_{k}$. It follows that $\operatorname{dim} f_{t}^{-1}(0)$ is constant over $R_{k}$. Therefore, to show our finiteness theorem, we can assume that $\operatorname{dim} f_{t}^{-1}(0)$ is constant over $J$.

(2) Subdivide $J$ into finite Nash manifolds $Q_{i}$ which are Nash diffeomorphic to open simplexes by Łojasiewicz's Semialgebraic Triangulation Theorem.

(3) Consider only Nash manifolds $Q_{i}$ of dimension $r$.

(4) Desingularise $F_{Q_{i}}^{-1}(0)$ in $N \times Q_{i}$ by the Desingularisation Theorem. 
(5) Using Semialgebraic Triangulation Theorem, Tarski-Seidenberg Theorem and Sard's Theorem again and again, we can subdivide $Q_{i}$ into finite Nash submanifolds $P_{i(j)}$ so that Nash Isotopy Lemma is applicable for $P_{i(j)}$ of dimension $r$. Then we can get a Nash trivial simultaneous resolution of $F_{P_{i(j)}}^{-1}(0)$ in $N \times P_{i(j)}$ over $P_{i(j)}$.

(6) The union $J_{1}$ of Nash manifolds $Q_{i}$ and $P_{i(j)}$ we removed in the above process is a semialgebraic subset of $J$ of dimension less than $r$.

Considering $J_{1}$ as $J$, repeat this procedure. Then we can get our finiteness theorem.

We next consider the local case. Let $J$ be a semialgebraic set in some Euclidean space, and let $f_{t}:\left(\mathbf{R}^{n}, 0\right) \rightarrow\left(\mathbf{R}^{k}, 0\right)(t \in J)$ be a Nash mapping. Assume that $F$ is a Nash mapping. Set

$$
K=\left\{t \in J \mid f_{t}^{-1}(0) \cap S\left(f_{t}\right) \subseteq\{0\} \text { as germs at } 0 \in \mathbf{R}^{n}\right\} .
$$

Then we can see that $K$ is a semialgebraic subset of $J$ ([FKS], Lemma 6.4).

We have the result corresponding to Theorem Ia in the local case.

TheOREM Ib ([K-3]). There exists a finite partition

$$
J=Q_{1} \cup \ldots \cup Q_{s} \cup Q_{s+1} \cup \ldots \cup Q_{u}
$$

with $K=Q_{1} \cup \ldots \cup Q_{s}$ and $J-K=Q_{s+1} \cup \ldots \cup Q_{u}$ which satisfies the following conditions:

(1) Each $Q_{i}$ is a Nash manifold which is Nash diffeomorphic to an open simplex in some Euclidean space.

(2) For each $i$, there is a Nash trivial simultaneous resolution $\Pi_{i}: \mathcal{M}_{i} \rightarrow \mathbf{R}^{n} \times Q_{i}$ of $F_{Q_{i}}^{-1}(0)$ in $\mathbf{R}^{n} \times Q_{i}$ over $Q_{i}$ around $\{0\} \times Q_{i}$.

In particular, for $1 \leq i \leq s$, this Nash trivialisation induces the semialgebraic trivialisation of $F_{Q_{i}}^{-1}(0)$ in $\mathbf{R}^{n} \times Q_{i}$ over $Q_{i}$ around $\{0\} \times Q_{i}$. (In this case, we say that $\left(\mathbf{R}^{n} \times Q_{i}, F_{Q_{i}}^{-1}(0)\right)$ admits a $\Pi_{i}$-Blow-Nash trivialisation along $Q_{i}$ around $\{0\} \times Q_{i}$. $)$

REmark 4.1. The latter half in Theorem $\mathrm{Ib}(2)$, namely, finiteness on Blow-Nash triviality for a family of Nash set-germs with isolated singularities was first proved in [FKS]. But it is natural to regard it as a part of the finiteness theorem above on Nash trivial simultaneous resolution.

When T. Fukui, M. Shiota and I wrote up [FKS], we did not know the desingularisation theorem in the Nash category. Therefore, after we proved a finiteness theorem on BlowNash triviality for a family of algebraic set-germs with isolated singularities, we reduced the Nash case to the algebraic case using the Artin-Mazur Theorem ([AM]). But, we need not make use of such a method, if we apply Theorem 2.1 directly.

When we apply Nash Isotopy Lemma in Section 3 to show the finiteness theorems on the existence of Nash trivial simultaneous resolution, properness is important. In the compact case, properness is automatically satisfied. But in the local case, some device is necessary. A similar situation happens also in T. Fukuda [Fu]. He proved Local Isotopy Lemma without the assumption of properness. (Only the statement is written in $[\mathrm{Fu}]$, but the proof was given also in the first draft.) Here we do not try to show a similar kind of Local Isotopy Lemma in the Nash category. Instead, when we treat the local 
case, we consider the objects in a closed neighbourhood of $\Pi_{i}^{-1}\left(\{0\} \times Q_{i}\right)$ which is a Nash manifold with boundary. In order to apply our Nash Isotopy Lemma in the proof of Theorem Ib, as such a closed neighbourhood, we have to construct a Nash manifold whose boundary is transverse to the strict transform of $V_{i}=F_{Q_{i}}^{-1}(0)$, all exceptional divisors and their arbitrary intersection. For this purpose, we introduced the notion of uniformity of Milnor radius via desingularisation in [K-3], and first proved a finiteness theorem on it. In the case of isolated singularities, the situation is simple. In this case it suffices to construct a semialgebraic neighbourhood of $\{0\} \times Q_{i}$ in $\mathbf{R}^{n} \times Q_{i}$ with usual uniformity of Milnor radius. Then we may assume that the inverse of the neighbourhood by $\Pi_{i}$ is a Nash manifold whose boundary is transverse to the strict transform of $V_{i}$ and does not intersect any exceptional divisor. An inequality of Thom's type is a useful tool to show a finiteness theorem on uniformity of Milnor radius in the case of isolated singularities. In fact, we used the inequality to show the latter half of Theorem $\operatorname{Ib}(2)$ in $[\mathrm{FKS}]$. For the detail of an inequality of Thom's type, see K. Bekka and S. Koike [BK].

5. Problems and partial results. In the previous section, we described finiteness theorems on the existence of Nash trivial simultaneous resolution in the global compact case and in the local case. Consecutively, we treat finiteness in the cases. As stated above, when the zero-sets of Nash mappings have isolated singularities, a Nash isomorphism upstairs automatically induces a $t$-level preserving semialgebraic homeomorphism downstairs trivialising $F_{Q_{i}}^{-1}(0)$. Therefore we got a Blow-Nash triviality. We consider a similar problem in the case of non-isolated singularities.

Problem 1. Does a finiteness theorem hold on Blow-Nash triviality for $\left\{\left(N, f_{t}^{-1}(0)\right)\right\}$ (or $\left\{\left(\mathbf{R}^{n}, f_{t}^{-1}(0)\right)\right\}$ ) in the case of non-isolated singularities, too?

The problem is not easy. In this case, we have to show Nash-semialgebraic triviality of each resolution mapping $\Pi_{i}$ as a family of Nash mappings. But we do not have any method to show such a triviality in general. In addition, local topological moduli appear in a family of polynomial map-germs $\left\{f_{t}:\left(\mathbf{R}^{n}, 0\right) \rightarrow\left(\mathbf{R}^{k}, 0\right)\right\}, n \geq 3, k \geq 2$, as stated in the introduction. Taking these facts into consideration, we pose the following weakened problem:

Problem 1-1. Does a finiteness theorem hold on Blow-semialgebraic triviality for $\left\{\left(N, f_{t}^{-1}(0)\right)\right\}$ (or $\left.\left\{\left(\mathbf{R}^{n}, f_{t}^{-1}(0)\right)\right\}\right)$ in the case of non-isolated singularities?

Blow-semialgebraic triviality means that there are a Nash simultaneous resolution $\Pi_{i}$ of $F_{Q_{i}}^{-1}(0)$ and a semialgebraic homeomorphism upstairs which induces a $t$-level preserving semialgebraic homeomorphism downstairs trivialising $\left(N \times Q_{i}, F_{Q_{i}}^{-1}(0)\right)$ (or $\left.\left(\mathbf{R}^{n} \times Q_{i}, F_{Q_{i}}^{-1}(0)\right)\right)$. Concerning this problem, we have some partial results.

Let $N$ be a compact Nash manifold of dimension less than or equal to 3 , and let $J$ be a semialgebraic set in some Euclidean space. Let $f_{t}: N \rightarrow \mathbf{R}^{k}(t \in J)$ be a Nash mapping. Assume that $F$ is a Nash mapping and $f_{t}^{-1}(0) \cap S\left(f_{t}\right)$ is not isolated for $t \in J$.

We have the following finiteness theorem on Blow-semialgebraic triviality. 
TheOREM IIa ([K-3]). There exists a finite partition $J=Q_{1} \cup \ldots \cup Q_{u}$ which satisfies the following conditions:

(1) Each $Q_{i}$ is a Nash manifold which is Nash diffeomorphic to an open simplex in some Euclidean space.

(2) For each $i$, there is a Nash simultaneous resolution $\Pi_{i}: \mathcal{M}_{i} \rightarrow N \times Q_{i}$ of $F_{Q_{i}}^{-1}(0)$ in $N \times Q_{i}$ over $Q_{i}$ such that $\left(N \times Q_{i}, F_{Q_{i}}^{-1}(0)\right)$ admits a $\Pi_{i}$-Blow-semialgebraic trivialisation along $Q_{i}$.

REMARK 5.1. Let $q: N \times J \rightarrow J$ be the canonical projection, and let $V_{i}=\left\{F_{Q_{i}}=0\right\}$. If $\operatorname{dim}\left(S\left(V_{i}\right) \cap q^{-1}(t)\right) \leq 0$ for any $t \in Q_{i}$, then subdividing $Q_{i}$ into finitely many Nash manifolds if necessary, $\left(N \times Q_{i}, F_{Q_{i}}^{-1}(0)\right)$ admits a $\Pi_{i}$-Blow-Nash trivialisation along $Q_{i}$. Here $S\left(V_{i}\right)$ denotes the singular points set of $V_{i}$ as a Nash variety, and $\operatorname{dim}(\emptyset)=-1$. Therefore in the case where $\operatorname{dim} N \leq 2,\left(N \times Q_{i}, F_{Q_{i}}^{-1}(0)\right)$ admits a $\Pi_{i}$-Blow-Nash trivialisation along $Q_{i}$ for $1 \leq i \leq u$.

We can show the theorem above using the semialgebraic versions of Thom's 1st and 2nd Isotopy Lemmas proved by M. Shiota [S-4]. We first make some remarks on the Isotopy Lemmas. The original Thom's Isotopy Lemmas ([T-1], [T-2], [T-3]) were proved in the $C^{2}$ category that is topological triviality follows from some regularity conditions (e.g. the Whitney $(b)$-regularity, the Thom $\left(a_{f}\right)$-regularity) on $C^{2}$ stratified sets and mappings. For the detailed proofs, see J. Mather [Mat] or C. G. Gibson, K. Wirthmüller, A. du Plessis and E. J. N. Looijenga [GWPL]. The semialgebraic versions were also proved under the same assumptions not only in the $C^{2}$ Nash category but also in the $C^{1}$ Nash category. But the proofs of the former are simpler than those of the latter. We use the Isotopy Lemmas in the $C^{2}$ Nash category. We make one more remark. If we replace the assumption of the $\left(a_{F}\right)$-regularity by the assumption of the $\left(a_{f_{t}}\right)$-regularity for any $t \in Q$, the semialgebraic version of Thom's 2nd Isotopy Lemma still holds after taking a finite subdivision of the parameter space $Q([\mathrm{~K}-3])$.

We describe the gist of the proof of Theorem IIa. By Theorem Ia, a finiteness theorem holds on the existence of Nash simultaneous resolution $\Pi_{i}: \mathcal{M}_{i} \rightarrow N \times Q_{i}$ of $F_{Q_{i}}^{-1}(0)$ in $N \times Q_{i}$ over $Q_{i}$. A pinch map is a typical example as a non-Thom mapping. Therefore we cannot apply the semialgebraic version of Thom's 2nd Isotopy Lemma directly to this $\Pi_{i}$. But taking a finite subdivision of $Q_{i}$ if necessary, we can construct $C^{\omega}$ Whitney stratifications $\mathcal{S}\left(\mathcal{M}_{i}\right)$ of $\mathcal{M}_{i}$ compatible with the strict transform of $F_{Q_{i}}^{-1}(0)$, exceptional divisors and their intersections and $\mathcal{S}\left(N \times Q_{i}\right)$ of $N \times Q_{i}$ compatible with their images by $\Pi_{i}$ so that the 2nd Isotopy Lemma (with the $\left(a_{f_{t}}\right)$-regularity for $\left.t \in Q_{i}\right)$ is applicable to the restriction of $\Pi_{i}$ to the union $\mathcal{D}$ of exceptional divisors. Therefore it follows that $\left.\Pi_{i}\right|_{\mathcal{D}}: \mathcal{D} \rightarrow$ $N \times Q_{i}$ is semialgebraically trivial over $Q_{i}$ (taking a finite subdivision of $Q_{i}$ if necessary). Let $q: N \times Q_{i} \rightarrow Q_{i}$ be the canonical projection. Then $\left.q \circ \Pi_{i}\right|_{\mathcal{D}}: \mathcal{D} \rightarrow Q_{i}$ is semialgebraically trivial over $Q_{i}$. By the semialgebraic version of Thom's 1st Isotopy Lemma, we can extend the semialgebraic triviality of $\left.q \circ \Pi_{i}\right|_{\mathcal{D}}$ over $Q_{i}$ to the semialgebraic one of $q \circ \Pi_{i}$ over $Q_{i}$. Since $\Pi_{i}: \mathcal{M}_{i} \rightarrow N \times Q_{i}$ is an isomorphism outside $\mathcal{D}$, the extended semialgebraic trivialisation of $q \circ \Pi_{i}$ induces a semialgebraic one of $\Pi_{i}$ over $Q_{i}$. We can show Blowsemialgebraic triviality in this way, using a combination of 2 nd and 1st Isotopy Lemmas. 
Let $N$ be a compact Nash manifold of dimension bigger than 3 , and let $J$ and $f_{t}$ : $N \rightarrow \mathbf{R}^{k}(t \in J)$ be the same as in Theorem IIa. We further assume that $\operatorname{dim} f_{t}^{-1}(0) \leq 2$ for $t \in J$. Then, using an argument similar to the above, we can show:

COROLlaRY IIIa. There exists a finite partition $J=Q_{1} \cup \ldots \cup Q_{u}$ which satisfies the following conditions:

(1) Each $Q_{i}$ is a Nash manifold which is Nash diffeomorphic to an open simplex in some Euclidean space.

(2) For each $i$, there is a Nash simultaneous resolution $\Pi_{i}: \mathcal{M}_{i} \rightarrow N \times Q_{i}$ of $F_{Q_{i}}^{-1}(0)$ in $N \times Q_{i}$ over $Q_{i}$ such that $F_{Q_{i}}^{-1}(0)$ admits a $\Pi_{i}$-Blow-semialgebraic trivialisation along $Q_{i}$. Namely, there is a semialgebraic trivialisation of the strict transform of $F_{Q_{i}}^{-1}(0)$ by $\Pi_{i}$ which induces a semialgebraic one of $F_{Q_{i}}^{-1}(0)$ over $Q_{i}$.

Let $J$ be a semialgebraic set in some Euclidean space, and let $f_{t}:\left(\mathbf{R}^{n}, 0\right) \rightarrow\left(\mathbf{R}^{k}, 0\right)$ $(t \in J)$ be a Nash mapping. Assume that $F$ is a Nash mapping and $f_{t}^{-1}(0) \cap S\left(f_{t}\right)$ is not contained in $\{0\}$ as germs at $0 \in \mathbf{R}^{n}$ for $t \in J$.

The results corresponding to Theorem IIa, Remark 5.1 and Corollary IIIa hold in the local case. In the case where $n \leq 3$, we have

TheOREM IIb ([K-3]). There exists a finite partition $J=Q_{1} \cup \ldots \cup Q_{u}$ which satisfies the following conditions:

(1) Each $Q_{i}$ is a Nash manifold which is Nash diffeomorphic to an open simplex in some Euclidean space.

(2) For each $i$, there is a Nash simultaneous resolution $\Pi_{i}: \mathcal{M}_{i} \rightarrow \mathbf{R}^{n} \times Q_{i}$ of $F_{Q_{i}}^{-1}(0)$ in $\mathbf{R}^{n} \times Q_{i}$ over $Q_{i}$ around $\{0\} \times Q_{i}$ such that $\left(\mathbf{R}^{n} \times Q_{i}, F_{Q_{i}}^{-1}(0)\right)$ admits a $\Pi_{i}$-Blow-semialgebraic trivialisation along $Q_{i}$ around $\{0\} \times Q_{i}$.

Assume that $n>3$ and $\operatorname{dim} f_{t}^{-1}(0) \leq 2$ for $t \in J$. Then we have

COROLLARY IIIb. There exists a finite partition $J=Q_{1} \cup \ldots \cup Q_{u}$ which satisfies the following conditions:

(1) Each $Q_{i}$ is a Nash manifold which is Nash diffeomorphic to an open simplex in some Euclidean space.

(2) For each $i$, there is a Nash simultaneous resolution $\Pi_{i}: \mathcal{M}_{i} \rightarrow \mathbf{R}^{n} \times Q_{i}$ of $F_{Q_{i}}^{-1}(0)$ in $\mathbf{R}^{n} \times Q_{i}$ over $Q_{i}$ around $\{0\} \times Q_{i}$ such that $F_{Q_{i}}^{-1}(0)$ admits a $\Pi_{i}$-Blow-semialgebraic trivialisation along $Q_{i}$ around $\{0\} \times Q_{i}$.

Concerning the corollaries above, we have the following problem:

Problem 1-2. Does a finiteness theorem hold on Blow-semialgebraic triviality for $\left\{f_{t}^{-1}(0)\right\}$ in the case of non-isolated singularities?

6. List of finiteness properties. We give the list of our results on finiteness properties. In this section we describe only the global compact case, but we have a similar list also in the local case.

Let $N$ be a compact Nash manifold of dimension $n$, and let $J$ be a semialgebraic set in some Euclidean space. Let $f_{t}: N \rightarrow \mathbf{R}^{k}(t \in J)$ be a Nash mapping. Assume that $F$ is 
a Nash mapping. As stated in the outline of the proof of Theorem Ia, when we consider our finiteness problem, we may assume from the beginning that $\operatorname{dim} f_{t}^{-1}(0)$ is constant over $J$. Then we can summarise our results more explicitly as follows:

\begin{tabular}{|c|c|c|c|}
\hline & $\operatorname{dim} f_{t}^{-1}(0)$ & $f_{t}^{-1}(0) \cap S\left(f_{t}\right)$ isolated $(=\emptyset)$ & $f_{t}^{-1}(0) \cap S\left(f_{t}\right)$ non-isolated \\
\hline \multirow{5}{*}{$\begin{array}{l}\text { Existence of } \\
\text { Nash trivial } \\
\text { simultaneous } \\
\text { resolution }\end{array}$} & 0 & $\begin{array}{l}\text { Nash triviality for } \\
\left\{\left(N, f_{t}^{-1}(0)\right)\right\}\end{array}$ & This case never happens \\
\hline & 1 & \multirow{4}{*}{$\begin{array}{l}\text { Blow-Nash triviality for } \\
\text { (Nash triviality for) } \\
\left\{\left(N, f_{t}^{-1}(0)\right)\right\}\end{array}$} & $\begin{array}{l}\text { Blow-Nash triviality } \\
\text { for }\left\{\left(N, f_{t}^{-1}(0)\right)\right\}\end{array}$ \\
\hline & 2 & & $\begin{array}{l}n=3 \text { : Blow-semialgebraic } \\
\text { triviality for }\left\{\left(N, f_{t}^{-1}(0)\right)\right\} \\
n \geq 4 \text { : Blow-semialgebraic } \\
\text { triviality for }\left\{f_{t}^{-1}(0)\right\}\end{array}$ \\
\hline & 3 & & A result in the algebraic case \\
\hline & $\geq 4$ & & Fukui's observation \\
\hline
\end{tabular}

The list shows on which triviality a finiteness theorem holds. For instance, it shows in the middle row that if $f_{t}^{-1}(0) \cap S\left(f_{t}\right)$ is isolated (resp. empty), a finiteness theorem holds on Blow-Nash triviality (resp. Nash triviality) for $\left\{\left(N, f_{t}^{-1}(0)\right)\right\}$ in the case where $\operatorname{dim} f_{t}^{-1}(0) \geq 1$.

In the former sections, we did not refer to any result in the case where $f_{t}^{-1}(0) \cap S\left(f_{t}\right)$ is non-isolated and $\operatorname{dim} f_{t}^{-1}(0) \geq 3$. We have some result related to Problem $1-2$ in the case where $\operatorname{dim} f_{t}^{-1}(0)=3$. But we do not have any positive result in the case where $\operatorname{dim} f_{t}^{-1}(0) \geq 4$. Recently, T. Fukui $([\mathrm{F}-2])$ gave an interesting observation in this case. We shall describe the result in the case where $\operatorname{dim} f_{t}^{-1}(0) \geq 3$ and the observation of Fukui in the next section.

7. Some remarks in the higher-dimensional case. We first describe a result concerning Problem 1-2 in the algebraic case. Let $N$ be a compact affine nonsingular algebraic variety or $\mathbf{R}^{n}$, and let $J$ be an affine algebraic variety. Let $f_{t}: N \rightarrow \mathbf{R}^{k}(t \in J)$ be a polynomial mapping such that $\operatorname{dim} f_{t}^{-1}(0) \leq 3$ for $t \in J$. Assume that $F$ is a polynomial mapping. Then we have the following finiteness theorem.

TheOREM IV ([K-4]). There exists a finite partition $J=Q_{1} \cup \ldots \cup Q_{u}$ which satisfies the following conditions:

(1) Each $Q_{i}$ is a Nash manifold which is Nash diffeomorphic to an open simplex in some Euclidean space.

(2) For each $i$, there is an algebraic simultaneous resolution $\Pi_{i}: \mathcal{M}_{i} \rightarrow N \times Q_{i}$ of $F_{Q_{i}}^{-1}(0)$ in $N \times Q_{i}$ over $Q_{i}$ such that $F_{Q_{i}}^{-1}(0)$ admits a $\Pi_{i}$-Blow-semialgebraic trivialisation along $Q_{i}$.

We can show this theorem using a similar argument as the proof of Corollary IIIa with Sabbah's arguments on "Morphismes stratifiés sans éclatement" ([Sa-1], [Sa-2]) based on the flattening theorem of Hironaka $([\mathrm{H}-2],[\mathrm{H}-4])$. The details will be written in the forthcoming paper. 
We next describe Fukui's observation. Let us recall the Nakai family.

ExAmple $7.1([\mathrm{~N}])$. Let $f_{e}=\left(f_{1 e}, f_{2 e}\right):\left(\mathbf{R}^{3}, 0\right) \rightarrow\left(\mathbf{R}^{2}, 0\right)$ be a polynomial mapping defined by

$$
\begin{aligned}
& f_{1 e}(x, y, z)=\left(e_{1} x-y\right)\left(e_{2} x-y\right)\left(e_{3} x-y\right), \\
& f_{2 e}(x, y, z)=\left(e_{4} x-y\right)\left(e_{5} x-y\right)\left(e_{6} x-y\right) z
\end{aligned}
$$

where $e=\left(e_{1}, e_{2}, e_{3}, e_{4}, e_{5}, e_{6}\right) \in \mathbf{R}^{6}$ with $e_{1}<e_{2}<e_{3}<e_{4}<e_{5}<e_{6}$. Then local topological moduli appear in the family $\left\{f_{e}\right\}$.

T. Fukui gave the following observation.

OBservation $7.2([\mathrm{~F}-2])$. There are a family of algebraic sets $V_{t} \subset \mathbf{R}^{6}$ with $\operatorname{dim} V_{t}=4$ and a simultaneous resolution $\left\{\pi_{t}: \mathcal{M}_{t} \rightarrow \mathbf{R}^{6}\right.$ of $\left.V_{t}\right\}$ such that $\left\{\left.\pi_{t}\right|_{V_{t}^{\prime} \cap E_{t}}\right\}$ at some point $P$ is the Nakai family. Here $V_{t}^{\prime}$ is the strict transform of $V_{t}$ by $\pi_{t}$ and $E_{t}$ is an exceptional divisor containing $P$. In addition, there is a natural identification of $\left(V_{t}^{\prime} \cap E_{t}, P\right)$ with $\left(\mathbf{R}^{3}, 0\right)$ for each $t$, and $\mathbf{R}^{2}=\mathbf{R}^{2} \times\{(0,0,0,0)\} \subset \mathbf{R}^{6}$.

The observation above means that in the case where $f_{t}^{-1}(0) \cap S\left(f_{t}\right)$ is non-isolated and $\operatorname{dim} f_{t}^{-1}(0) \geq 4$, we cannot show a finiteness theorem even on blow-semialgebraic triviality for a family of zero-sets $\left\{f_{t}^{-1}(0)\right\}$ using just the existence of a desingularisation of $F^{-1}(0)$. But the desingularisation procedure in Theorem 2.1 is canonical. Therefore there is a possibility that finiteness holds on semialgebraic triviality for the family of Hironaka's resolution mappings (or Bierstone-Milman's resolution mappings). In fact, Fukui's choice of centres of the blowings-up is essentially different from the canonical one in Theorem 2.1. At present, we do not know the answer to the finiteness problem in this case.

Generalising Fukui's argument, K. Bekka and S. Koike showed the following:

ASSERTION 7.3. Let $f=\left(f_{1}, \ldots, f_{p}\right):\left(\mathbf{R}^{n}, 0\right) \rightarrow\left(\mathbf{R}^{p}, 0\right)$ be a polynomial mapping such that $f_{j}$ is not identically zero for $1 \leq j \leq p$, and let $m$ be an arbitrary positive integer with $m \geq n+p+1$. Then there are an algebraic variety $V$ in $\mathbf{R}^{m}$ and a blowingup $\Pi: \mathcal{M} \rightarrow \mathbf{R}^{m}$ with an $(m-n-1)$-dimensional centre such that $\left.\Pi\right|_{V^{\prime} \cap E}$ at some point $P \in \mathcal{M}$ is $f$.

Proof. Let the source space $\mathbf{R}^{n}$ of $f$ be the $\left(x_{m-n+1}, \ldots, x_{m}\right)$-space, and let

$$
f_{j}\left(x_{m-n+1}, \ldots, x_{m}\right)=\sum_{\alpha} a_{\alpha}^{(j)} x_{m-n+1}^{\alpha_{m-n+1}} \cdots x_{m}^{\alpha_{m}}, 1 \leq j \leq p
$$

where $\alpha=\left(\alpha_{m-n+1}, \ldots, \alpha_{m}\right)$. Set $|\alpha|=\alpha_{m-n+1}+\ldots+\alpha_{m}$, and let $d_{j}$ be the degree of $f_{j}, 1 \leq j \leq p$. Define $g_{j}:\left(\mathbf{R}^{n+1}, 0\right) \rightarrow(\mathbf{R}, 0), 1 \leq j \leq p$, by

$$
g_{j}\left(x_{m-n}, x_{m-n+1}, \ldots, x_{m}\right)=\sum_{\alpha} a_{\alpha}^{(j)} x_{m-n}^{d_{j}-|\alpha|} x_{m-n+1}^{\alpha_{m-n+1}} \cdots x_{m}^{\alpha_{m}} .
$$

Then each $g_{j}$ is a homogeneous polynomial of degree $d_{j}$.

Let $k=\max \left(d_{1}, \ldots, d_{p}\right)$, and let $x=\left(x_{m-n}, x_{m-n+1}, \ldots, x_{m}\right)$. Define $F:\left(\mathbf{R}^{m}, 0\right) \rightarrow$ $(\mathbf{R}, 0)$ by

$$
\begin{aligned}
F\left(x_{1}, \ldots, x_{m}\right)=x_{m-n}^{2\left(k-d_{1}\right)}\left(x_{1} x_{m-n}^{d_{1}}-g_{1}(x)\right)^{2}+\ldots & +x_{m-n}^{2\left(k-d_{p}\right)}\left(x_{p} x_{m-n}^{d_{p}}-g_{p}(x)\right)^{2} \\
& +x_{m-n}^{2 k}\left(x_{p+1}^{2}+\ldots+x_{m-n-1}^{2}\right) .
\end{aligned}
$$


Then $V=F^{-1}(0)$ is an algebraic variety in $\mathbf{R}^{m}$ of dimension greater than or equal to $m-n-1$.

Consider the blowing-up $\Pi: \mathcal{M} \rightarrow \mathbf{R}^{m}$ with centre $C=\left\{x_{m-n}=\ldots=x_{m}=0\right\}$. Using a suitable chart $\mathcal{U}$, we can express $\Pi$ as follows:

$$
\begin{gathered}
x_{1}=u_{1}, \ldots, x_{m-n-1}=u_{m-n-1} \\
x_{m-n}=u_{m-n}, x_{m-n+1}=u_{m-n} u_{m-n+1}, \ldots, x_{m}=u_{m-n} u_{m} .
\end{gathered}
$$

Then we have

$$
\begin{aligned}
F \circ \Pi\left(u_{1}, \ldots, u_{m}\right)=u_{m-n}^{2 k} & \left\{\left(u_{1}-f_{1}\left(u_{m-n+1}, \ldots, u_{m}\right)\right)^{2}+\ldots\right. \\
& \left.+\left(u_{p}-f_{p}\left(u_{m-n+1}, \ldots, u_{m}\right)\right)^{2}+u_{p+1}^{2}+\ldots+u_{m-n-1}^{2}\right\} .
\end{aligned}
$$

In $\mathcal{U}$, the exceptional divisor $E$ is $\left\{u_{m-n}=0\right\}$ and the strict transform $V^{\prime}$ of $V$ by $\Pi$ is $\left\{u_{1}=f_{1}\left(u_{m-n+1}, \ldots, u_{m}\right), \ldots, u_{p}=f_{p}\left(u_{m-n+1}, \ldots, u_{m}\right), u_{p+1}=\ldots=u_{m-n-1}=0\right\}$.

The strict transform is smooth in $\mathcal{U}$ and of dimension $n+1$. Since $E$ is transverse to $V^{\prime}$, $V^{\prime} \cap E$ is a smooth submanifold of $\mathcal{U}$ and can be identified with the $\left(u_{m-n+1}, \ldots, u_{m}\right)$ space. Then in $\mathcal{U},\left.\Pi\right|_{V^{\prime} \cap E}: \mathbf{R}^{n} \rightarrow \mathbf{R}^{p} \times\{(0, \ldots, 0)\}$ is given by

$$
x_{1}=f_{1}\left(u_{m-n+1}, \ldots, u_{m}\right), \ldots, x_{p}=f_{p}\left(u_{m-n+1}, \ldots, u_{m}\right) .
$$

Thus we can regard $\left.\Pi\right|_{V^{\prime} \cap E}$ at $0 \in \mathcal{U}$ as $f$.

REMARK 7.4. In the proof of the assertion, while $V^{\prime}$ is smooth in $\mathcal{U}$, it may have singularities at $\infty$ outside $\mathcal{U}$. Assume that $\operatorname{dim} V=n+1\left(=\operatorname{dim} V^{\prime}\right)$ and $\operatorname{dim} C \leq n$. Then, using the desingularisation theorem of Hironaka ([H-1]), we can desingularise $V^{\prime}$ without touching a neighbourhood of $0 \in V^{\prime} \cap E \cap \mathcal{U}$. Namely, we can get the statement of the assertion for a desingularisation $\Pi$ of $V$ not just a blowing-up $\Pi$ of $\mathbf{R}^{m}$.

In the case of Fukui's observation, $n=3, p=2, m=n+p+1=6, \operatorname{dim} V=n+1=4$, and $\operatorname{dim} C=2$. It satisfies our assumption.

Concerning Fukui's observation and the remark above, we have the following natural problem:

Problem 2. Let $f=\left(f_{1}, \ldots, f_{p}\right):\left(\mathbf{R}^{n}, 0\right) \rightarrow\left(\mathbf{R}^{p}, 0\right)$ be a polynomial mapping such that $f_{j}$ is not identically zero for $1 \leq j \leq p$. Are there an algebraic variety $V$ in some Euclidean space $\mathbf{R}^{m}$ and a desingularisation $\Pi: \mathcal{M} \rightarrow \mathbf{R}^{m}$ of $V$ in $\mathbf{R}^{m}$ such that $\left.\Pi\right|_{V^{\prime} \cap E}$ at some point $P \in \mathcal{M}$ is $f$ ? Here $V^{\prime}$ is the strict transform of $V$ by $\Pi$ and $E$ is the union of exceptional divisors.

From the construction of $F$ in Assertion 7.3 and Remark 7.4, it follows that the answer to Problem 2 is yes in the function case that is $p=1$.

Supplement. The Briançon-Speder family $\left\{f_{t}\right\}([\mathrm{BS}])$ is well-known as an example that even in the complex case topological triviality does not always imply the Whitney (b)-regularity. In the real case the family of weighted homogeneous polynomial function-germs with isolated singularities admits a blow-analytic trivialisation (T. Fukui [F-1], T. Fukui and L. Paunescu [FP]), as conjectured by T.-C. Kuo. Therefore the real Briançon-Speder family is blow-analytically trivial. On the other hand, I proved in [K-1] 
that the blow-analytic triviality does not preserve the tangency of analytic arcs in $f_{t}^{-1}(0)$. As a result, blow-analytic equivalence for analytic function-germs does not always imply Lipschitz one for analytic set-germs. Then, looking for stronger triviality than topological one for the zero-sets of the real Briançon-Speder family, I introduced the notion of П-modified Nash triviality for a family of zero-sets of weighted homogeneous polynomial mappings using a weighted double oriented blowing-up $\Pi$ in the sense of H. Hironaka [H-4], and proved that the family is $\Pi$-modified Nash trivial if the zero-sets have isolated singularities ([K-2]). In the homogeneous case, the modified Nash triviality induces a Blow-Nash triviality in our sense. Concerning the modified Nash triviality, we have started to consider finiteness problems on Blow-Nash triviality for a family of Nash sets. In a global meaning, our finiteness problems are, of course, deeply related to equisingularity problems originated in O. Zariski [Z-1], [Z-2], [Z-3], [Z-4].

Acknowledgements. I wish to thank Karim Bekka, Toshizumi Fukui, Tzee-Char Kuo, Tadeusz Mostowski and Adam Parusiński for helpful discussions about Assertion 7.3, and to Professor Heisuke Hironaka for giving me some useful comments.

\section{References}

[A] K. Aoki, On topological types of polynomial map germs of plane to plane, Mem. School Sci. Engrg. Waseda Univ. 44 (1980), 133-156.

[AM] M. Artin, B. Mazur, On periodic points, Ann. of Math. (2) 81 (1965), 82-99.

[BK] K. Bekka, S. Koike, The Kuo condition, an inequality of Thom's type and (c)-regularity, Topology 37 (1998), 45-62.

[BM-1] E. Bierstone, P. D. Milman, Uniformization of analytic spaces, J. Amer. Math. Soc. 2 (1989), 801-836.

[BM-2] E. Bierstone, P. D. Milman, A simple constructive proof of canonical resolution of singularities, in: Effective Methods in Algebraic Geometry, Progr. Math. 94, Birkhäuser, Boston, 1991, 11-30.

[BM-3] E. Bierstone, P. D. Milman, Canonical desingularization in characteristic zero by blowing up the maximum strata of a local invariant, Invent. Math. 128 (1997), 207-302.

[BS] J. Briançon, J. P. Speder, La trivialité topologique n'implique pas les conditions de Whitney, C. R. Acad. Sci. Paris Sér. A-B 280 (1975), A365-A367.

[CS-1] M. Coste, M. Shiota, Nash triviality in families of Nash manifolds, Invent. Math. 108 (1992), 349-368.

[CS-2] M. Coste, M. Shiota, Thom's first isotopy lemma: a semialgebraic version with uniform bounds, in: Real Analytic and Algebraic Geometry (Trento, 1992), Walter de Gruyter, Berlin, 1995, 83-101.

[Fu] T. Fukuda, Types topologiques des polynômes, Inst. Hautes Études Sci. Publ. Math. 46 (1976), 87-106.

[F-1] T. Fukui, Modified analytic trivialization via weighted blowing up, J. Math. Soc. Japan 44 (1992), 455-459.

[F-2] T. Fukui, Introduction to toric modifications with an application to real singularities, Sūrikaisekikenkyūsho Kōkyūroku 1122 (2000), 96-114 (in Japanese). 
[FKK] T. Fukui, S. Koike, T.-C. Kuo, Blow-analytic equisingularities, properties, problems and progress, in: Real Analytic and Algebraic Singularities, Pitman Res. Notes Math. Ser. 381, Longman, Harlow, 1998, 8-29.

[FKS] T. Fukui, S. Koike, M. Shiota, Modified Nash triviality of a family of zero-sets of real polynomial mappings, Ann. Inst. Fourier (Grenoble) 48 (1998), 1395-1440.

[FP] T. Fukui, L. Paunescu, Modified analytic trivialization for weighted homogeneous function-germs, J. Math. Soc. Japan 52 (2000), 433-446.

[GWPL] C. G. Gibson, K. Wirthmüller, A. A. du Plessis, E. J. N. Looijenga, Topological Stability of Smooth Mappings, Lecture Notes in Math. 552, Springer, Berlin, 1976.

[Ha] R. M. Hardt, Semi-algebraic local triviality in semi-algebraic mappings, Amer. J. Math. 102 (1980), 291-302.

[H-1] H. Hironaka, Resolution of singularities of an algebraic variety over a field of characteristic zero: I, II, Ann. of Math. (2) 79 (1964), 109-203, 205-326.

[H-2] H. Hironaka, Flattening theorem in complex-analytic geometry, Amer. J. Math. 97 (1975), 503-547.

[H-3] H. Hironaka, Idealistic exponents of singularity, in: Algebraic Geometry (J. J. Sylvester Sympos.), Johns Hopkins Univ. Press, Baltimore, 1977, 52-125.

[H-4] H. Hironaka, Stratification and flatness, in: Real and Complex Singularities (Oslo, 1976), Sijthoff and Noordhoff, Alphen aan den Rijn, 1977, 199-265.

[K-1] S. Koike, On strong $C^{0}$-equivalence of real analytic functions, J. Math. Soc. Japan 45 (1993), 313-320.

[K-2] S. Koike, Modified Nash triviality theorem for a family of zero-sets of weighted homogeneous polynomial mappings, J. Math. Soc. Japan 49 (1997), 617-631.

[K-3] S. Koike, Nash trivial simultaneous resolution for a family of zero-sets of Nash mappings, Math. Z. 234 (2000), 313-338.

[K-4] S. Koike, Blow-semialgebraic triviality for a family of 3-dimensional algebraic sets, in preparation.

[Ku-1] T.-C. Kuo, Une classification des singularités réelles, C. R. Acad. Sci. Paris Sér. A-B 288 (1979), A809-A812.

[Ku-2] T.-C. Kuo, The modified analytic trivialization of singularities, J. Math. Soc. Japan 32 (1980), 605-614.

[Ku-3] T.-C. Kuo, On classification of real singularities, Invent. Math. 82 (1985), 257-262.

[L-1] S. Łojasiewicz, Triangulation of semi-analytic sets, Ann. Scuola Norm. Sup. Pisa (3) 18 (1964), 449-474.

[L-2] S. Łojasiewicz, Ensembles semi-analytiques, Inst. Hautes Études Sci. Lecture Note, 1965 .

[M] B. Malgrange, Ideals of Differentiable Functions, Oxford University Press, London, 1967.

[Mat] J. Mather, Notes on Topological Stability, Lecture Notes, Harvard University, 1970.

[N] I. Nakai, On topological types of polynomial mappings, Topology 23 (1984), 45-66.

[Sa-1] C. Sabbah, Le type topologique éclaté d'une application analytique, in: Singularities, Part 2 (Arcata, 1981), Proc. Symp. Pure Math. 40, Amer. Math. Soc., Providence, 1983, 433-440.

[Sa-2] C. Sabbah, Morphismes analytiques stratifiés sans éclatement et cycles évanescents, Astérisque 101-102 (1983), 286-319.

[Se] A. Seidenberg, A new decision method for elementary algebra, Ann. of Math. (2) 60 (1954), 365-374. 
[S-1] M. Shiota, Classification of Nash manifolds, Ann. Inst. Fourier (Grenoble) 33 (1983), 209-232.

[S-2] M. Shiota, Approximation theorems for Nash mappings and Nash manifolds, Trans. Amer. Math. Soc. 293 (1986), 319-337.

[S-3] M. Shiota, Nash Manifolds, Lecture Notes in Math. 1269, Springer, Berlin, 1987.

[S-4] M. Shiota, Geometry of Subanalytic and Semialgebraic Sets, Progr. Math. 150, Birkhäuser, Boston, 1997.

[T-1] R. Thom, Local topological properties of differentiable mappings, in: Differential Analysis (Bombay Colloq.), Oxford Univ. Press, London, 1964, 191-202.

[T-2] R. Thom, Propriétés différentielles locales des ensembles analytiques, Séminaire Bourbaki 9, Exp. 281 (1964-65), Soc. Math. France, Paris, 1995, 69-80.

[T-3] R. Thom, Ensembles et morphismes stratifiés, Bull. Amer. Math. Soc. 75 (1969), 240-284.

[V] A. N. Varchenko, Theorems on the topological equisingularity of families of algebraic varieties and families of polynomial mappings, Izv. Akad. Nauk SSSR Ser. Mat. 36 (1972), 957-1019 (in Russian).

[W] H. Whitney, Local topological properties of analytic varieties, in: Differential and Combinatorial Topology (A Symposium in Honor of M. Morse), Princeton Univ. Press, Princeton, 1965, 205-244.

[Z-1] O. Zariski, Studies in equisingularity I. Equivalent singularities of plane algebroid curves, Amer. J. Math. 87 (1965), 507-536.

[Z-2] O. Zariski, Studies in equisingularity II. Equisingularity in codimension 1 (and characteristic zero), Amer. J. Math. 87 (1965), 972-1006.

[Z-3] O. Zariski, Studies in equisingularity III. Saturation of local rings and equisingularity, Amer. J. Math. 90 (1968), 961-1032.

[Z-4] O. Zariski, Some open questions in the theory of singularities, Bull. Amer. Math. Soc. 77 (1971), 481-491. 